\title{
Feasibility and predictors of change of narrative exposure therapy for displaced populations: a repeated measures design
}

Rina S. Ghafoerkhan ${ }^{1,2^{*}}$ (D), Henriette E. van Heemstra ${ }^{1,2^{*}+}$, Willem F. Scholte ${ }^{1,3,4}$, Joriene R. J. van der Kolk ${ }^{4,5}$, Jackie June F. ter Heide ${ }^{1}$, Simone M. de la Rie ${ }^{1}$, Linda M. Verhaak ${ }^{1}$ Evelien Snippe ${ }^{6}$ and Paul A. Boelen ${ }^{1,2}$

\begin{abstract}
Background: Displaced victims of interpersonal violence, such as refugees, asylum seekers, and victims of sexual exploitation, are growing in numbers and are often suffering from a post-traumatic stress disorder (PTSD). At the same time, these victims are known to benefit less from trauma-focused therapy (TFT) and to be less compliant to treatment. The objective of this paper is to describe the rationale and research protocol of an ongoing trial that aims to evaluate different variables that might influence the feasibility of TFT for the study population. Specifically, perceived daily stress, emotion regulation, and mood are investigated as predictors of change in PTSD symptoms during a trauma-focused therapy (narrative exposure therapy (NET)). The feasibility of administering measures tapping these constructs repeatedly during treatment will also be evaluated.
\end{abstract}

Methods/design: Using an observational treatment design, 80 displaced victims of interpersonal violence will be measured before, during, and after partaking in NET. Several questionnaires tapping PTSD plus the aforementioned possible predictors of PTSD change will be administered: Post-traumatic Stress Disorder Checklist-5, Perceived Stress Scale, Difficulties in Emotion Regulation Scale-18 (pre-test, post-test, and follow-up),subscale impulsivity of the Difficulties in Emotion Regulation Scale-18, Perceived Stress Scale short version, Primary Care Post-traumatic Stress Disorder and a single Mood item (each session). Multilevel modelling will be used to examine the relation between the possible predictors and treatment outcome.

Discussion: The present study is the first to examine the interplay of facilitating and interfering factors possibly impacting treatment feasibility and effectiveness in displaced victims of interpersonal violence with PTSD receiving NET, using repeated measures. The current study can help to improve future treatment based on individual characteristics.

Trial registration: Netherlands Trial Register: NTR7353, retrospectively registered. Date of registration: July 11, 2018.

Keywords: Refugees, Human trafficking, Sexual exploitation, Post-traumatic stress disorder, Narrative exposure therapy, Treatment response, Feasibility

\footnotetext{
* Correspondence: r.ghafoerkhan@arq.org; j.van.heemstra@arq.org

${ }^{\dagger}$ Rina S. Ghafoerkhan and Henriëtte E. van Heemstra contributed equally to this work.

${ }^{1}$ ARQ National Psychotrauma Centre, Nienoord 5, 1112 XE Diemen, The Netherlands

Full list of author information is available at the end of the article
}

(c) The Author(s). 2020 Open Access This article is licensed under a Creative Commons Attribution 4.0 International License, which permits use, sharing, adaptation, distribution and reproduction in any medium or format, as long as you give appropriate credit to the original author(s) and the source, provide a link to the Creative Commons licence, and indicate if changes were made. The images or other third party material in this article are included in the article's Creative Commons licence, unless indicated otherwise in a credit line to the material. If material is not included in the article's Creative Commons licence and your intended use is not permitted by statutory regulation or exceeds the permitted use, you will need to obtain permission directly from the copyright holder. To view a copy of this licence, visit http://creativecommons.org/licenses/by/4.0/ The Creative Commons Public Domain Dedication waiver (http://creativecommons.org/publicdomain/zero/1.0/) applies to the data made available in this article, unless otherwise stated in a credit line to the data. 


\section{Background}

Worldwide, there is an increase in the number of victims of interpersonal violence who are forced to leave their home country [1]. When entering a host country, these displaced persons are usually referred to as either 'refugees', 'asylum seekers', 'trafficked human beings', 'illegal immigrants' or 'undocumented people', depending on their trauma background and legal status. Mostly these groups overlap in their experience of forced migration, their marginalized social position, and the challenges they encounter. In this paper, we will therefore collectively refer to all such groups as 'displaced people'.

As a result of assorted traumatic events, like war- and conflict-related violence, sexual violence and exploitation, many of these displaced people suffer from a posttraumatic stress disorder (PTSD) [2-5]. PTSD symptoms cause a great burden, render people unable to engage in daily activities and put them at risk for re-victimization [6]. Displaced people suffering from PTSD could therefore benefit from trauma-focused therapy (TFT). TFT is a psychological intervention aiming to decrease PTSD symptoms by fostering the processing of traumatic memories.

Scientific research into TFT for displaced people is scarce. Studies into TFT for non-refugee traumatized populations show higher effects $(d=1.08-1.40)$ [7], than for refugee populations $(g=.25-1.01)$ [8]. Moreover, the feasibility of evidence-based therapies within the refugee population is complicated by post-migration obstacles [9] which can result in low treatment compliance and completion levels. Most likely, the primary focus on PTSD fails to address the broader challenges faced by displaced people [10]. Therefore, more insight is needed into factors affecting treatment response during TFT and conditions for recovery. Identifying factors that undermine the feasibility of TFT for displaced people may help to refine the timing and focus of interventions, and thus improve treatment response.

One key factor that potentially undermines the feasibility of treatment is daily stressors, given their proven association with PTSD symptoms [11-13]. Ongoing daily stressors arising from the immigration process, loss of social network, and impaired functioning resulting from PTSD symptoms negatively impact mental health among the study population [13-16]. The burden caused by ongoing daily stressors may impact one's cognitive functioning in several ways [17]. For example, by occupying the working memory [18], and thereby reducing the cognitive resources needed to process traumatic events.

While there is evidence for the negative impact of daily stressors and perceived daily stress on treatment for displaced populations, the few present studies have yielded ambiguous results. One study affirmed the negative impact of lack of social support, a daily stressor, when present at the start of treatment on treatment outcome [19]. However, a study where clinician-rated daily stressors during treatment could not establish such an impact on treatment [20]. These limited and contradictive findings illustrate the need for more rigorous research on the relation between daily stressors and treatment response within the study population. No study to date has explored the impact of experienced daily stressors while taking part in TFT in the study population.

Another main factor likely to affect the feasibility of TFT for displaced people is emotion regulation, such as insight into, control over, and awareness of one's emotions (e.g., [21, 22]). Several studies have indicated that emotion dysregulation mediates between traumatic events and the development of PTSD in different groups (e.g., [23]), including traumatized refugees [24]. Emotion dysregulation has been identified as a consequence of trauma and emotional impulsivity in particular as a predictor of future (re)victimization $[25,26]$. For TFT to be feasibly applied, a person must be able to stay within a dynamic 'window of tolerance', a range of affect that can be regulated at that point in time [27]. Consequently, it is expected that the level of emotion regulation at baseline and improved control over one's emotions during treatment are both prerequisites for reducing PTSD symptoms [28]. No study to date has looked into the interplay between emotion regulation and PTSD symptoms while taking part in TFT in the study population.

A final main factor that may affect treatment feasibility is depressed mood, which can be measured as a proxy for depression [29]. Among resettled refugees, the comorbidity rate for PTSD with depression is $44 \%$; for depression with PTSD, it is 71\% [2]. Previous research on TFT shows that higher baseline levels of depression predict poorer treatment response in displaced individuals [30] and therewith undermine the feasibility of treatment. Meanwhile, TFT has proven effectiveness in reducing symptoms of depression in the study population [31]. Yet, insight in the interaction between depressed mood and PTSD during treatment is currently lacking.

In an umbrella review of prevalence and intervention studies on common mental disorders in asylum seekers and refugees, Turrini et al. [32] found that narrative exposure therapy (NET) was the best-supported TFT for reducing PTSD symptoms. In a meta-analysis of NET [33], it was found that NET is effective in reducing PTSD and depression symptoms across diverse, predominantly war-affected refugee populations [34]. Since it is a first-choice treatment for the study population, NET was chosen as the TFT applied in the present study [33].

As outlined above, several factors (e.g. PTSD, perceived daily stress, emotion dysregulation and mood) tend to impact treatment feasibility. However, their 
interplay during TFT has not yet been examined. Insight in the feasibility of measuring this interplay in a diverse group of displaced individuals following NET is yet to be established. To best of our knowledge, there are no prior treatment studies for which weekly repeated measures have been carried out within the target population. Additionally, the practical execution of examining the interrelatedness between different parameters (e.g. PTSD, perceived daily stress, emotion dysregulation and mood) is based on questionnaires that are partly adapted for the current study (see 'Methods' section). Consequently, their feasibility within the target population has not been objectified yet.

In the present paper, the feasibility, rationale and protocol of an ongoing trial are described. Specifically, we aim to identify relevant predictors of PTSD symptom change during and after NET in 80 displaced victims of interpersonal violence. The primary hypotheses of the study are (1) it is feasible to administer highly repeated measures, within a diverse group of displaced persons. (2) High perceived daily stress, emotion dysregulation, and low mood at baseline and during NET predict higher drop-out, higher no-show and poorer treatment response of NET (i.e. less PTSD symptom reduction), thus undermining the feasibility of NET; (3) Reduction in perceived daily stress and improvement in emotion regulation and mood during NET are associated with concurrent reductions in PTSD symptoms during NET; (4) Change in perceived daily stress, emotion dysregulation, and mood during NET predict subsequent changes in PTSD symptoms at later stages of NET. Furthermore, the study aims to establish whether NET contributes to positive aspects of mental health.

\section{Methods}

This study follows a repeated measures observational design with baseline assessments, repeated measures over the course of treatment and post-treatment assessments. Twelve to sixteen sessions of NET will be provided to all patients included in the study. Various questionnaires will be administered at baseline (T0, pre-treatment), 1 week after NET completion (T1, post-treatment) and 6 weeks after NET completion (T2, follow-up). In addition, assessments to measure potential processes of change will be performed at the start of each NET session. See Fig. 1 for a detailed overview of the planned design and administered measures.

NET will be provided by trained psychologists, psychotherapists, medical doctors and psychiatrists. Psychologists and master's level psychology students will perform assessments for the study. All involved professionals have extensive experience in working with migrants (in a culturally sensitive manner) and interpreters.

\section{Participants}

The study will take place at an outpatient clinic specialized in mental health care for refugees, asylum seekers, victims of sexual exploitation and otherwise traumatized populations in the Capital region in the Netherlands. The study aims to include 80 participants, in order to detect medium to small effect sizes [35]. Patients are referred to the clinic by a general practitioner or by a partnering social welfare organization. Patients' background/nationality is diverse; most originate from West Africa, the Middle East and Eastern Europe. Their legal status varies from having obtained a residence permit or even Dutch citizenship to illegal residence in the country, and they may reside in asylum seekers centres, specialized shelters for victims of sexual exploitation, governmental shelters for illegal persons or live independently.

\section{Inclusion criteria}

Patients will be included in the study if they are displaced victims of interpersonal violence, aged 18 years or

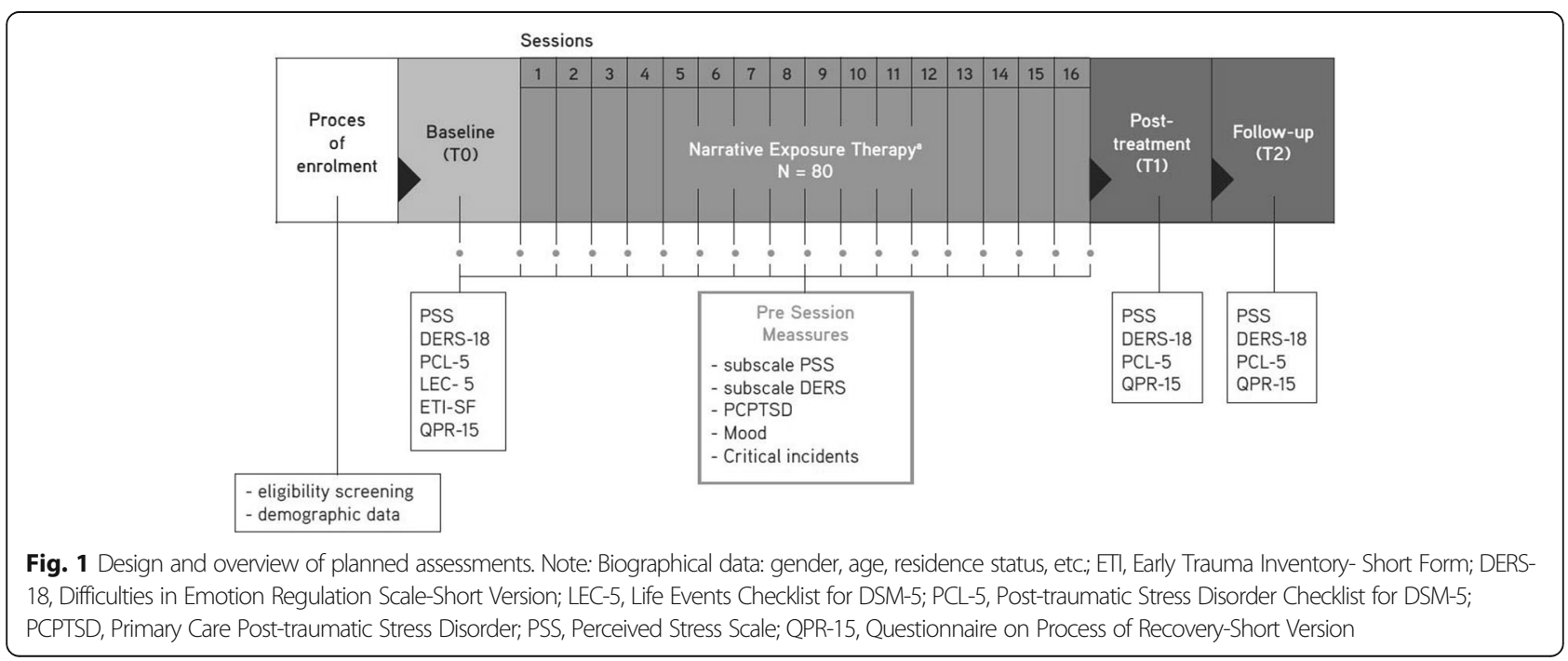


older, if they have PTSD as a primary diagnosis established by a psychiatrist or clinical psychologist during intake, if individual NET in an outpatient setting has been indicated for them and if they are cognitively able to give informed consent to participate in the study.

\section{Exclusion criteria}

Patients will be excluded from the study if they display signs of an acute crisis, such as acute suicidality or acute severe psychosis, or suffer from persistent substance abuse. These exclusion criteria are applied because of their expected disturbing influence on the adherence and/or completion of NET.

\section{Recruitment}

This is an ongoing treatment trial. The enrolment period is planned to run for 2.5 years from February 2018 to January 2021. Following multi-disciplinary clinical assessment and evaluation, the diagnosis and treatment indication will be discussed with patients. Those who are eligible for enrolment will be informed about the study and will be invited to participate. This eligibility will be considered as broadly as possible within the inclusion criteria to allow for a representative sample of displaced victims of interpersonal violence. Information about the study aims and objectives, guarantee of anonymization of data and the fact that participants are free to terminate participation at any moment will be given orally and provided on paper through an information leaflet. After, intake patients are placed on a waiting list for NET, ranging from 1 to 4 months. When patients from the waiting list are invited to start NET, they will once again be informed about the study orally and on paper. Patients will be asked to consider participation over a minimum period of 3 days. Afterwards, they will be contacted by phone or face-to face and given ample opportunity to ask additional questions. If they indicate willingness to participate in the study, an appointment is made to sign informed consent and conduct the baseline assessment (T0). Participants are invited to bring someone they trust to this meeting. Participants will be receiving a voucher of 10 euros after the follow-up measurement. If after the first year the aspired total number of participants seems unachievable, prolongation of the enrolment period and multicentre options will be explored after consultation with the involved medical ethical committee.

\section{Intervention}

\section{Narrative exposure therapy}

NET is an evidence-based short-term psychotherapy targeting PTSD symptoms, specifically appropriate for multiple traumas in divergent cultural settings. For the study population in question, the method is found to be feasible [36] and is considered the first choice TFT [33]. The
NET protocol includes 12-16 sessions of individual trauma focused exposure, performed weekly or twice a week by trained mental health professionals. Each session lasts $90-120 \mathrm{~min}$, depending on the content of the trauma which is targeted during the session, and the possible involvement of an interpreter. NET aims to create a chronological narrative of a patient's life story, including both traumatic and empowering memories. During each session, one or more significant memories (traumatic or empowering) are discussed in great detail. Imaginary exposure, meaning-making, and reprocessing are used to reduce PTSD symptoms. An account of each session is written down by the therapist, which will result in a patient's biography when the therapy has been completed.

\section{Co-interventions before and during treatment}

When participants cannot immediately start NET, there are no restrictions in psychological or pharmacological interventions received during their waiting period, applied in accordance with the national guidelines. Such interventions can be indicated either for symptom management or as a preparation of individual TFT. In addition, individual sessions can be provided in case of (imminent) crisis. From the start of NET onwards, however, no other modules will be provided, unless in case of acute crisis in which the safety of a participant is endangered or he/she is about to harm others. Previously started pharmacological interventions can be continued during NET; however, no new pharmacological interventions will be started during NET.

\section{Discontinuation of the intervention and drop-out}

Discontinuation of NET will take place if patients so wish or if multi-disciplinary clinical evaluation indicates that continuation is not in the best interest of the patient. If patients wish to stop participating in the study but wish to continue NET, this will be allowed. Patients will be considered dropouts when there are deviations from the NET treatment protocol for more than four consecutive sessions (i.e. no trauma-focused approach during the sessions or no-show), since in these cases the effectiveness of the treatment offered cannot be assured [34].

\section{Measurements \\ Perceived Stress Scale (PSS)—full scale administered pre- and post-treatment}

The PSS [37] has been developed to measure the perception of daily stress by assessing how unpredictable, uncontrollable and overloading patients experience daily life. With 10 items, thoughts and feelings and the evaluation of daily life in the last month are explored through a 5-point scale ranging from 0 to 4 . Mean scores will be calculated, ranging from 0 to 4 . Example item: 'In the 
last month, how often have you been upset because of something that happened unexpectedly?' Administration of the questionnaire takes approximately $10 \mathrm{~min}$. Acceptable psychometric properties of the instrument have been established [38].

\section{Subscale administered before each session}

The four items version of the PSS [39] will be administered at the start of each session. These items have proven sensitivity to short-term changes in stress [40]. For the purpose of this study, the indicated timespan was changed from 'In the last month' to 'In the last week' to match the other pre-sessions measures for possible predictors. An example item is 'In the last week, how often have you felt that things were going your way?' These items will be scored on a VAS-scale of exact $10 \mathrm{~cm}$ by placing a cross on a line from 0 (not at all) to 100 (completely). Mean scores will be calculated, ranging from 0 to 100 .

\section{Difficulties in Emotion Regulation Scale short version-18 (DERS)_full scale administered pre- and post-treatment}

The DERS was developed to measure emotion regulation. The DERS-18 comprises 18 items and uses a 5-point Likert scale ranging from 1 to 5 ; it has 6 subscales: nonacceptance of emotional responses, difficulty engaging in goal-directed behaviour, impulse control difficulties, lack of emotional awareness, limited access to emotion regulation strategies and a lack of emotional clarity. Mean scores will be calculated, ranging from 1 to 5 . Example item: 'I pay attention to how I feel'. Administration of the questionnaire takes approximately $10 \mathrm{~min}$. The DERS has high internal consistency, good test-retest reliability, moderate construct and predictive validity [41].

\section{Subscale 'impulsivity' administered before each session}

As a proxy of emotion regulation, the subscale 'Impulsivity' will be used, comprising three items from the 'Difficulties in Emotion Regulation Scale' (DERS-18; 40). For the purpose of this study, the phrase 'In the last week I have felt:' was added to match the other pre-session measures for possible predictors. Example item: 'When I am upset, I become out of control'. Items will be scored on a VASscale ranging from 0 (not at all) to 100 (completely). Mean scores will be calculated, ranging from 0 to 100 .

\section{PTSD Checklist for DSM-5 (PCL-5)—administered pre-and post-treatment}

The 20 item PCL- 5 is a self-report checklist which measures the presence and severity of the 20 DSM-5 symptoms of PTSD on a 5-point scale (0-4) (e.g. 'Trouble remembering important parts of the stressful experience'). Mean scores will be calculated, ranging from 0 to 4. It will be used to indicate PTSD symptom severity pre- and post-treatment. Administration of the questionnaire takes approximately $10 \mathrm{~min}$. The instrument has good psychometric quality [42].

\section{Primary Care Post-traumatic Stress Disorder (PCPTSD)_administered before each session}

Because a short version of the PCL- 5 is unavailable, the five-item Primary Care Post-traumatic Stress Disorder (PCPTSD) checklist was selected to increase the feasibility of the frequently repeated measurements. This questionnaire is used to measure PTSD symptoms [43]. For the purpose of this study the indicated timespan was changed from 'In the past month, have you' to 'In the last week' to match the other pre-session measures for possible predictors. An example item is: 'In the last week did you have nightmares about the event(s) or thoughts about the event(s) when you did not want to?' These items will be scored on a VAS-scale ranging from 0 (not at all) to 100 (extremely). The questionnaire has good psychometric qualities [41]. Mean scores will be calculated, ranging from 0 to 100 .

\section{Mood-measured before each session}

Mood will be measured using a validated single item measure [44]. For the purpose of this study, we have altered the item 'At the moment I feel' to 'In the last week I felt' to make the timespan congruent to the other presession measures for possible predictors. This mood item will be scored on a VAS-scale ranging from 0 (sad) to 100 (happy). Mean scores will be calculated, ranging from 0 to 100 .

\section{Critical incidents}

Before each therapy session, the client will be asked to report if any relevant personal circumstances have arisen since the last appointment.

\section{Other}

Biographical data of patients will be collected (i.e. gender, age, educational level, current residence, legal status, country of birth) to describe the study population.

\section{Questionnaire on Process of Recovery Short Version-15}

The QPR is a 5-point scale (0-4), self-report questionnaire that probes people's recovery and meaningful aspects in the recovery process (e.g., 'I feel able to take chances in life.'). The QPR-15 consists of 15 items. Mean scores will be calculated, ranging from 0 to 4 . The questionnaire has good psychometric properties, and has proven to be associated with quality of life, empowerment, and psychological wellbeing [45]. Administration of the questionnaire lasts approximately 20 minutes. The questionnaire has been selected because it represents psychological wellbeing beyond the scope of mental 
health symptoms. The QPR is reliable and valid, and has proven to be associated with quality of life, empowerment and psychological wellbeing [45].

\section{Early Trauma Inventory-short version}

The Early Trauma Inventory-short version (ETI-SF) was developed to determine potentially traumatic events before and after the age of 18 years old [46]. The ETI-SF comprises 27 items that assess physical, emotional, and sexual abuse. Items vary between open questions and multiple choice being answered with yes or no. The scale has a range from 0 to 29 , with higher scores referring to a higher number of traumatic experiences. Administration of the questionnaire lasts approximately $10 \mathrm{~min}$. Its reliability and validity are good [47]. The questionnaire has been selected to describe trauma-related features of the study population.

\section{Life Events Checklist (LEC-5)}

The LEC-5 comprises 17 multiple choice items on a 6point nominal scale (i.e., 'happened to me', 'witnessed it', 'learned about it', 'part of my job', 'not sure', 'doesn't apply'). The checklist aims to determine whether someone has ever been exposed to 16 events known to potentially result in PTSD or distress, and one additional event not captured in the first 16 items. In the last part of the LEC-5, respondents are asked which event he/she considers to have had the most impact, followed by 7 questions, both open- and multiple-choice questions, aimed at identifying the characteristics of this event. The scale has a range from 0 to 68 , with higher scores referring to a higher number of traumatic experiences. Administration of the questionnaire lasts approximately 20 min. The reliability of the LEC-5 is considered to be good [48]. The questionnaire was selected to describe trauma-related features of the study population.

\section{Data collection, management and analysis Procedure pre-session measures}

During baseline (T0) and at the start of each TFT session, the patient's fixed practitioner or a supervised master's level psychology student will administer the thirteen items selected to assess the four abovementioned possible predictors. To avoid an order effect, the sequence of the measures for possible predictors and the order of questions within every measure will change with every session. These measures will only be administered during the treatment period. They will be administered by pen and paper and are expected to take on average $10 \mathrm{~min}$. Questionnaires are translated from their original English version according a forward-backward procedure by two independent native speakers into Dutch, and by professional translators into Arabic,
French, Amharic and Tigrinya. Interpreters will assist for other languages.

\section{Procedure pre- and post-treatment}

During baseline (T0), post-treatment (T1) and follow-up (T2) measurements, the abovementioned set of questionnaires will be administered. See Fig. 1 for a detailed overview of the planned time points for assessments. These questionnaires will be administered digitally and are expected to take a maximum of $80 \mathrm{~min}$. Questionnaires are available in Dutch and English. Given the lack of computer skills and the often limited Dutch and/or English proficiency within the study population, a clinician and interpreter will be available for assistance. Measurements will take place at the treatment location; if preferred, this meeting will be combined with other appointments. After the last assessment, participants will receive a voucher of 10 euros.

\section{Statistical analysis}

First, multiple regression analyses and logistic regression analyses will be performed to examine whether high perceived daily stress, emotion dysregulation and low mood at baseline predict drop-out, no-show and treatment outcomes. Secondly, the concurrent association between each possible predictor measured prior to each session (i.e. perceived daily stress, emotion regulation and mood) and PTSD symptoms will be examined using bivariate (multilevel) growth modelling, as multiple observations of the predictor variables and PTSD symptoms (level 1) are nested within individuals (level 2) [49]. In order to infer that change in the predictor variables leads to change in PTSD symptoms, we will examine the timeline (i.e. temporal precedence) in two ways $[50,51]$. The dynamic (i.e. temporal) associations will be examined by estimating whether change in PTSD symptoms from the previous week to the current week $(\mathrm{t})$ can be predicted by change of a possible predictor at the previous week ( $t-1)$ using multilevel modelling. To examine the timeline of larger shifts instead of week-to-week changes, it will be examined whether the earliest significant decrease in mean levels of each possible predictor (improvement) occurs before the largest reduction in mean levels of PTSD symptoms.

\section{Discussion}

Among the growing number of forcibly displaced people worldwide, many are suffering from trauma-related mental health problems. As psychological treatments for displaced persons with PTSD appear to be less successful than for other populations, insight is needed in factors that affect the feasibility of these treatments. However, relevant research is lacking. The ongoing trial presented in this paper is the first to examine the 
interplay of factors for feasibility and effectiveness of in displaced victims of interpersonal violence receiving NET. More specifically, the impact of perceived daily stress, emotion dysregulation, and disturbed mood on PTSD symptom changes during TFT are examined.

The theoretical basis of NET has been well documented, and its effectiveness has been examined in various studies and contexts [33, 34, 36]. Yet, specific factors contributing to positive outcomes or constraining its feasibility are largely unidentified. In the current study, repeated measures will identify various constructs relevant for treatment feasibility and response of NET. Since, to our knowledge, this is the first study to use repeated measures within the target population, findings will additionally provide insight into the feasibility of this method for displaced populations.

As the method requires only minor adaptations to usual treatment proceedings and follows an observational design, participants can be included that might have to be excluded in more complex designs [52]. This allows for conclusions based on a sample representing the intended population with high external validity, which is exceptional for this specific group [53]. Although our design favours generalizability of the results, it may reduce the internal validity of the study; for instance, the present design does not allow for the examination of the extent to which observed changes in PTSD can be attributed to NET, but to factors such as natural recovery, bias or confounders. However, as we aim to study factors promoting or constraining treatment feasibility, a naturalistic design is the most ethical, feasible and time-efficient design to obtain answers to our research questions. Besides, the reduced internal validity is partially addressed by limiting the possibly confounding role of concurrent treatment.

Evidently, there are factors possibly impacting treatment response. For the purpose of this study, repeated measures were selected on the basis of relevant studies $[41,44]$ and clinical insights. Thus, the set of questions have been tailored to the specific characteristics of the study population. Conclusions drawn from the study therefore may hold relevant implications in clinical practice for displaced victims of interpersonal violence. Although the chosen subscales support the clinical validity of the study, the PTSD and emotion dysregulation subscales have not been validated as weekly repeated measures.

It is important to note that inclusion for the current study has already started; however, it is planned to run until early 2021. Critically, no data analysis has currently been performed yet.

We expect that the findings of this study will contribute to both the scientific and the clinical field. Identifying factors that limit treatment response may in turn inform the development of improved treatment modules that specifically address these blockages. The promotion of self-efficacy or problem-solving skills prior to NET, for example, may decrease perceived daily stress and its constraining effect on the feasibility of NET. Likewise, promoting emotion regulation prior to NET may intensify its potential supportive role for TFT. Additionally, if these factors have no predictive value for the course of PTSD during treatment, clinicians might be encouraged to offer TFT to patients despite the presence of daily stressors, emotion regulation difficulties and mood problems. By identifying predictors of treatment response, the current study can enable treatment indications to be tailored to individual characteristics.

Furthermore, exploring these predictors sheds a broader light on the consequences of trauma, beyond a narrow focus on PTSD, and may provide clues for a wider range of relevant treatment foci. In this way, this study responds to the ongoing debate on the emphasis focus of PTSD in designing treatments for displaced populations [10].

\section{Abbreviations \\ ETI: Early Trauma InventoryDERSDifficulties in Emotion Regulation Scale; LEC: Life Events ChecklistNETNarrative exposure therapy; PCL: PTSD Checklist for DSM-5; PCPTSD: Primary Care Post-traumatic Stress Disorder; PSS: Perceived Stress Scale; PTSD: Post-traumatic stress disorder; QPR: Questionnaire on Process of Recovery; TFT: Trauma-Focused Therapy}

\section{Acknowledgements}

We would like to thank our patients in advance, our clinical team members who helped to draft the study protocol and will carry out the treatments and our stakeholders. Also, we would like to thank Utrecht University, ARQ National Psychotrauma Centre for facilitating this study. Finally, we would like to thank Claudi Bockting, Anke Lahuis and Marieke van Gelderen for their advising roles in various stages of drafting the study protocol.

\section{Study status}

The treatment trial described in this paper has recently started and is ongoing. This article was first submitted on July 13,2019, and then resubmitted on August 26, 2020. The study started recruitment in March 2018, the first patient was included on March 30, 2018. Recruitment is expected to continue till January 2021 to reach the intended number of participants. No publications containing the results of this study have been published or submitted to any journal.

\section{Authors' contributions}

JVH, RG and WS initiated the study based on the identified clinical relevance. $\mathrm{JVH}$ and RG are the primary investigators of the study, designed the study and are the primary authors of the protocol as well as this manuscript. WS and $\mathrm{PB}$ are the primary supervisors and participated in the design of the study and helped to draft and revise the protocol and this manuscript. ES has participated in designing the study, helped to draft the protocol, has revised the manuscript and will be supervising the interpretation of data. LV, JvdK, FtH and SdIR are the clinical team leaders in the mental health care facilities in which the study is conducted, they have advised in the design of the study protocol and are involved in the data collection and monitoring of the study. All authors read and approved the final manuscript.

\section{Funding}

Partially funded by anonymous private fund. The study protocol did not undergo peer-review by this fund. The funder will not have the ultimate authority over any of the study activities. 


\section{Availability of data and materials}

The datasets generated and/or analyzed during the current study are not publicly available due to confidentiality of the patient data but are available from the corresponding author on reasonable request. Scientific papers will be written based on the collected data. For secondary data-analysis, the first authors can be contacted.

\section{Ethics approval and consent to participate}

Ethical approval for the study has been obtained from the Ethical Committee Psychology at the University of Leiden in the Netherlands (P17.270).

\section{Consent for publication}

Consent for publication of the collected data will be obtained from the participants.

\section{Competing interests}

The authors declare that they have no competing interests.

\begin{abstract}
Author details
${ }^{1}$ ARQ National Psychotrauma Centre, Nienoord 5, 1112 XE Diemen, The Netherlands. ${ }^{2}$ Department of Clinical Psychology, Utrecht University, Heidelberglaan 1, 3584 CS Utrecht, The Netherlands. ${ }^{3}$ Dept. of Psychiatry, Amsterdam UMC, University of Amsterdam, Meibergdreef 9, 1105 AZ Amsterdam, The Netherlands. ${ }^{4}$ Laguna Collective, Reigerstraat 16, 3816 AX Amersfoort, The Netherlands. ${ }^{5}$ Sinai Centrum, Arthur van Schendelstraat 800, 3511 ML Utrecht, The Netherlands. ${ }^{6}$ Department of Psychiatry, Interdisciplinary Center Psychopathology and Emotion Regulation, University of Groningen, University Medical Center Groningen, Hanzeplein 1, 9713 GZ Groningen, The Netherlands.
\end{abstract}

Received: 13 March 2020 Accepted: 4 May 2020

Published online: 21 May 2020

\section{References}

1. Global Trends. Forced displacement in 2016. Geneva: UNHCR; 19-6-2017. 71 p.

2. Fazel M, Wheeler J, Danesh J. Prevalence of serious mental disorder in 7000 refugees resettled in western countries: a systematic review. Lancet. 2005; 365(9467):1309-14. https://doi.org/10.1016/S0140-6736(05)61027-6.

3. Lindert J, von Ehrenstein OS, Priebe S, Mielck A, Brähler E. Depression and anxiety in labor migrants and refugees-a systematic review and metaanalysis. Soc Sci Med. 2009;69(2):246-57. https://doi.org/10.1016/j.socscimed 2009.04.032

4. Steel Z, Chey T, Silove D, Marnane C, Bryant RA, Van Ommeren M. Association of torture and other potentially traumatic events with mental health outcomes among populations exposed to mass conflict and displacement: a systematic review and meta-analysis. Jama. 2009;302(5):53749. https://doi.org/10.1001/jama.2009.1132.

5. Zimmerman C, Hossain M, Yun K, Roche B, Morison L, Watts C. Stolen smiles: a summary report on the physical and psychological health consequences of women and adolescents trafficked in Europe. Stolen smiles: a summary report on the physical and psychological health consequences of women and adolescents trafficked in Europe. 2006.

6. Risser HJ, Hetzel-Riggin MD, Thomsen CJ, McCanne TR. PTSD as a mediato of sexual revictimization: the role of reexperiencing, avoidance, and arousal symptoms. Journal of Traumatic Stress: Official Publication of The International Society for Traumatic Stress Studies. 2006;19(5):687-98. https:// doi.org/10.1002/jts.20156.

7. Cusack K, Jonas DE, Forneris CA, Wines C, Sonis J, Middleton JC, et al. Psychological treatments for adults with posttraumatic stress disorder: a systematic review and meta-analysis. Clin Psychol Rev. 2016;43:128-41. https://doi.org/10.1016/j.cpr.2015.10.003.

8. Lambert JE, Alhassoon OM. Trauma-focused therapy for refugees: metaanalytic findings. J Couns Psychol. 2015;62(1):28. https://doi.org/10.1037/ cou0000048.

9. Slobodin O, de Jong JT. Mental health interventions for traumatized asylum seekers and refugees: what do we know about their efficacy? Int J Soc Psychiatry. 2015;61(1):17-26. https://doi.org/10.1177/0020764014535752.

10. Miller KE, Rasmussen A. Mental health and armed conflict: the importance of distinguishing between war exposure and other sources of adversity: a response to Neuner. Soc Sci Med. 2010;71(8):1385-9. https://doi.org/10. 1016/j.socscimed.2009.09.029.

11. Leon KA, Hyre AD, Ompad D, DeSalvo KB, Muntner P. Perceived stress among a workforce 6 months following hurricane Katrina. Soc Psychiatry Psychiatr Epidemiol. 2007;42(12):1005-11. https://doi.org/10.1007/s00127007-0260-6.

12. McAlonan GM, Lee AM, Cheung V, Cheung C, Tsang KW, Sham PC, et al. Immediate and sustained psychological impact of an emerging infectious disease outbreak on health care workers. Can J Psychiatry. 2007:52(4):241-7. https://doi.org/10.1177/ 070674370705200406.

13. Chu T, Keller AS, Rasmussen A. Effects of post-migration factors on PTSD outcomes among immigrant survivors of political violence. J Immigr Minor Health. 2013;15(5):890-7. https://doi.org/10.1007/s10903-012-9696-1.

14. Laban CJ, Gernaat HB, Komproe IH, van der Tweel I, De Jong JT. Postmigration living problems and common psychiatric disorders in Iraqi asylum seekers in the Netherlands. J Nerv Ment Dis. 2005;193(12):825-32. https://doi.org/10.1097/01.nmd.0000188977.44657.1d.

15. Li SS, Liddell BJ, Nickerson A. The relationship between post-migration stress and psychological disorders in refugees and asylum seekers. Current psychiatry reports. 2016;18(9):82. https://doi.org/10.1007/s11920-016-0723-0.

16. Priebe S, Giacco D, El-Nagib R. Public health aspects of mental health among migrants and refugees: a review of the evidence on mental health care for refugees, asylum seekers and irregular migrants in the WHO European Region. 2016.

17. McEwen BS, Sapolsky RM. Stress and cognitive function. Curr Opin Neurobiol. 1995;5(2):205-16. https://doi.org/10.1016/0959-4388(95)80028-X.

18. Luettgau, L., Schlagenhauf, F., \& Sjoerds, Z. (2018). Acute and past subjective stress influence working memory and related neural substrates. Psychoneuroendocrinology, 96, doi.org/10.1016/j.psyneuen.2018.05.036.

19. Buhman C, Mortensen E, Lundstrøm S, Ryberg J, Nordentoft M, Ekstrøm M. Symptoms, quality of life and level of functioning of traumatized refugees at Psychiatric Trauma Clinic in Copenhagen. Tor J Theol. 2018;24(1):15 doi. org/10/7146/torture.v24i1.109717

20. Bruhn M, Rees S, Mohsin M, Silove D, Carlsson J. The range and impact of postmigration stressors during treatment of trauma-affected refugees. J Nerv Ment Dis. 2018;206(1):61-8. https://doi.org/10.1097/NMD. 0000000000000774.

21. Goldin PR, Lee I, Ziv M, Jazaieri H, Heimberg RG, Gross JJ. Trajectories of change in emotion regulation and social anxiety during cognitivebehavioral therapy for social anxiety disorder. Behav Res Ther. 2014;56:7-15. https://doi.org/10.1016/j.brat.2014.02.005.

22. Gratz KL, Bardeen JR, Levy R, Dixon-Gordon KL, Tull MT. Mechanisms of change in an emotion regulation group therapy for deliberate self-harm among women with borderline personality disorder. Behav Res Ther. 2015; 65:29-35. https://doi.org/10.1016/j.brat.2014.12.005.

23. Messman-Moore TL, Bhuptani PH. A review of the long-term impact of child maltreatment on posttraumatic stress disorder and its comorbidities: an emotion dysregulation perspective. Clin Psychol Sci Pract. 2017;24(2):154-69. https://doi.org/10.1111/cpsp.12193.

24. Nickerson A, Bryant RA, Schnyder U, Schick M, Mueller J, Morina N. Emotion dysregulation mediates the relationship between trauma exposure, post-migration living difficulties and psychological outcomes in traumatized refugees. J Affect Disord. 2015;173:185-92. https:/doi.org/10.1016/j.jad.2014.10.043.

25. Cloitre M, Scarvalone P, Difede J. Posttraumatic stress disorder, self-and interpersonal dysfunction among sexually retraumatized women. J Trauma Stress. 1997;10(3):437-52. https://doi.org/10.1002/jts.2490100309.

26. Messman-Moore TL, Walsh KL, DiLillo D. Emotion dysregulation and risky sexual behavior in revictimization. Child Abuse Negl. 2010;34(12):967-76. https://doi.org/10.1016/ j.chiabu.2010.06.004

27. Siegel DJ. The developing mind (Vol. 296). New York: Guilford Press; 1999.

28. Hien DA, Lopez-Castro T, Papini S, Gorman B, Ruglass LM. Emotion dysregulation moderates the effect of cognitive behavior therapy with prolonged exposure for co-occurring PTSD and substance use disorders. J Anxiety Disord. 2017;52:53-61. https://doi.org/10.1016/j.janxdis.2017.10.003.

29. Aguilera A, Schueller SM, Leykin Y. Daily mood ratings via text message as a proxy for clinic based depression assessment. J Affect Disord. 2015;175:4714. https://doi.org/10.1016/ j.jad.2015.01.033.

30. Haagen JF, Ter Heide FJJ, Mooren TM, Knipscheer JW, Kleber RJ. Predicting post-traumatic stress disorder treatment response in refugees: multilevel analysis. Br J Clin Psychol. 2017:56(1):69-83. https:// doi.org/10.1111/bjc.12121 
31. Nosè M, Ballette F, Bighelli I, Turrini G, Purgato M, Tol W, et al. Psychosocial interventions for post-traumatic stress disorder in refugees and asylum seekers resettled in high-income countries: systematic review and metaanalysis. PLoS One. 2017;12(2):e0171030. https://doi.org/10.1371/journal/ pone.0171030

32. Turrini G, Purgato M, Ballette F, Nosè M, Ostuzzi G, Barbui C. Common mental disorders in asylum seekers and refugees: umbrella review of prevalence and intervention studies. Int J Ment Heal Syst. 2017;11(1):51. https://doi.org/10.3280/RSF2017-003004.

33. Lely JC, Smid GE, Jongedijk RA, W. Knipscheer J, Kleber RJ. The effectiveness of narrative exposure therapy: a review, meta-analysis and meta-regression analysis. Eur J Psychotraumatol. 2019;10(1):1550344.

34. Schauer M, Schauer M, Neuner F, Elbert T. Narrative exposure therapy: a shortterm treatment for traumatic stress disorders: Hogrefe Publishing; 2011.

35. Cohen J. Statistical power analysis for the behavioral sciences 2 nd edn. Hillsdale: Erlbaum Associates; 1988.

36. Halvorsen $\lrcorner \varnothing$, Stenmark $H$. Narrative exposure therapy for posttraumatic stress disorder in tortured refugees: a preliminary uncontrolled trial. Scand J Psychol. 2010;51(6):495-502. https://doi.org/10.1111/j.1467-9450. 2010.00821.x

37. Cohen S, Kamarck T, Mermelstein R. A global measure of perceived stress. J Health Soc Behav. 1983:385-96.

38. Cohen S, Kamarck T, Mermelstein R. Perceived stress scale. Measuring stress: a guide for health and social scientists. 1994:235-83.

39. Lee $\mathrm{E}-\mathrm{H}$. Review of the psychometric evidence of the perceived stress scale. Asian nursing research. 2012;6(4):121-7. https://doi.org/10.1016/j.anr.2012.08.004.

40. Baer RA, Carmody J, Hunsinger M. Weekly change in mindfulness and perceived stress in a mindfulness-based stress reduction program. J Clin Psychol. 2012;68(7):755-65. https://doi.org/10.1002/jclp.21865.

41. Victor SE, Klonsky ED. Validation of a brief version of the difficulties in emotion regulation scale (DERS-18) in five samples. J Psychopathol Behav Assess. 2016;38(4):582-9. https://doi.org/10.1007/s10862-016-9547-9.

42. Blevins CA, Weathers FW, Davis MT, Witte TK, Domino JL. The posttraumatic stress disorder checklist for DSM-5 (PCL-5): development and initial psychometric evaluation. J Trauma Stress. 2015;28(6):489-98. https://doi.org/ 10.1002/jts.22059.

43. Prins A, Bovin MJ, Smolenski DJ, Marx BP, Kimerling R, Jenkins-Guarnieri MA, et al. The primary care PTSD screen for DSM-5 (PC-PTSD-5): development and evaluation within a veteran primary care sample. J Gen Intern Med. 2016:31(10):1206-11. https://doi.org/10.1007/s11606-016-3703-5.

44. van Rijsbergen GD, Bockting CL, Berking M, Koeter MW, Schene AH. Can a one-item mood scale do the trick? Predicting relapse over 5.5-years in recurrent depression. PLoS One. 2012;7(10):e46796. https://doi.org/10.1371/ journal.pone.0046796.

45. Law H, Neil ST, Dunn G, Morrison AP. Psychometric properties of the questionnaire about the process of recovery (QPR). Schizophr Res. 2014; 156(2-3):184-9. https://doi.org/10.1016/j.schres.2014.04.011.

46. Bernstein DP, Stein JA, Newcomb MD, Walker E, Pogge D, Ahluvalia T, et al. Development and validation of a brief screening version of the Childhood Trauma Questionnaire. Child Abuse Negl. 2003;27(2):169-90. https://doi.org/ 10.1016/S0145-2134(02)00541-0

47. Bremner JD, Bolus R, Mayer EA. Psychometric properties of the early trauma inventory-self report. J Nerv Ment Dis. 2007;195(3):211. https://doi.org/10. 1097/ 01.nmd.0000243824.84651.6c.

48. Gray MJ, Litz BT, Hsu JL, Lombardo TW. Psychometric properties of the life events checklist. Assessment. 2004;11(4):330-341. DOl: 0.1177/ 1073191104269954.

49. Snijders TA, Bosker RJ. Modeled variance in two-level models. Sociol Methods Res. 1994;22(3):342-63. https://doi.org/10.1177/ 0049124194022003004

50. Kazdin AE, Nock MK. Delineating mechanisms of change in child and adolescent therapy: methodological issues and research recommendations. J Child Psychol Psychiatry. 2003;44(8):1116-29. https://doi.org/10.1111/14697610.00195.

51. Kraemer HC, Yesavage JA, Taylor JL, Kupfer D. How can we learn about developmental processes from cross-sectional studies, or can we? Am J Psychiatr. 2000;157(2):163-71.

52. Ross S, Grant A, Counsell C, Gillespie W, Russell I, Prescott R. Barriers to participation in randomised controlled trials: a systematic review. J Clin Epidemiol. 1999;52(12):1143-56. https://doi.org/10.1016/S08954356(99)00141-9
53. Enticott JC, Shawyer F, Vasi S, Buck K, Cheng I-H, Russell G, et al. A systematic review of studies with a representative sample of refugees and asylum seekers living in the community for participation in mental health research. BMC Med Res Methodol. 2017;17(1):37. https://doi.org/10.1186/ s12874-017-0312-X.

\section{Publisher's Note}

Springer Nature remains neutral with regard to jurisdictional claims in published maps and institutional affiliations.
Ready to submit your research? Choose BMC and benefit from:

- fast, convenient online submission

- thorough peer review by experienced researchers in your field

- rapid publication on acceptance

- support for research data, including large and complex data types

- gold Open Access which fosters wider collaboration and increased citations

- maximum visibility for your research: over $100 \mathrm{M}$ website views per year

At $\mathrm{BMC}$, research is always in progress.

Learn more biomedcentral.com/submissions 